\title{
Context-aware Collection, Decision, and Distribution (C2D2) Engine for Multi-Dimensional Adaptation in Vehicular Networks
}

\author{
Joseph Camp \\ Electrical Engineering \\ Southern Methodist \\ University \\ camp@smu.edu
}

\author{
Onur Altintas \\ Toyota InfoTechnology \\ Center, Co. Ltd. \\ Tokyo, Japan \\ onur@jp.toyota- \\ itc.com
}

\author{
Rama Vuyyuru \\ Toyota InfoTechnology \\ Center, U.S.A. Inc. \\ Mountain View, CA \\ rama@us.toyota- \\ itc.com
}

\author{
Dinesh Rajan \\ Electrical Engineering \\ Southern Methodist \\ University \\ rajand@smu.edu
}

\begin{abstract}
Wireless vehicular networks have highly complex and dynamic channel state leading to challenging environments to maintain connectivity and/or achieve high throughput while satisfying latency requirements of diverse vehicular applications. Adaptation over a large parameter space such as multiple frequency bands, novel modulation and coding schemes, and routing protocols is important in achieving good performance in these settings. Vehicles now include a plethora of sensors which can be used to establish a clearer notion of the environmental context. However, while it is well understood that wireless performance greatly depends on this contextual information, protocols that leverage this information to improve wireless performance have yet to be fully developed. In this work, we lay a foundation for developing context-aware intelligence to interface with existing adaptation protocols at multiple layers of the network stack. The core of this system consists of a context-aware collection, decision, and distribution (C2D2) engine. We give a brief overview of the architecture, design, and operation of the C2D2 engine.
\end{abstract}

\section{Categories and Subject Descriptors}

C.2.1 [Computer-Communication Networks]: Network Architecture and Design - Wireless Communication.

\section{General Terms}

Algorithms, Measurement, Performance, Experimentation.

\section{Keywords}

Vehicular Networks, Context Awareness, Wireless, Adaptation.

\section{INTRODUCTION}

Wireless vehicular networks have a high degree of channel variability, requiring fast convergence of adaptation algorithms to both remain connected and have good performance. While it is known that topological and environmental features of a region

Permission to make digital or hard copies of all or part of this work for personal or classroom use is granted without fee provided that copies are not made or distributed for profit or commercial advantage and that copies bear this notice and the full citation on the first page. To copy otherwise, or republish, to post on servers or to redistribute to lists, requires prior specific permission and/or a fee.

VANET'11, September 23, 2011, Las Vegas, Nevada, USA

Copyright 2011 ACM 978-1-4503-0869-4/11/09...\$10.00. have direct impact on wireless performance, the protocol stack for wireless networks has yet to fully exploit the available contextual data. Instead, protocols have either directly measured channel measurements or inferred the channel state via packet losses. The former technique can lead to an accurate estimate of the current channel state if variations are slow but, by definition, use a view of the channel which occurred in the past and can incur system overhead and delays. The latter technique also suffers from overhead and delays but additionally can lead to inaccurate channel state conclusions. A third option is emerging based on the increased sensing and storage ability of the wireless hardware-to cache wireless performance based on context to allow wireless protocols to quickly converge to high performance and automatically adjust upon detection of contextual changes.

In this work, we describe a context-aware collection, decision, and distribution (C2D2) engine for improved adaptation in all layers of the protocol stack for vehicular network applications. The C2D2 engine will collect sensor and network performance data to map wireless behavior to environmental context. Both a locally cached and global database will be built to store this training information where a particular data point is stored if the context is sufficiently different form prior training data. The collected data is also used by a decision engine to identify possible adaptation choices for each layer of the network stack that satisfy quality-ofservice metrics specified by the application. The decision subsystem will then distribute these available options to the existing network protocols, presenting a reduced searchable space for adaptation and allowing legacy operation. There are a number of intriguing questions that we seek to answer with the C2D2 engine. These questions include:

- Which context data leads to the most improved performance and which network-stack layer does context impact most? - When should certain context data be ignored or prioritized? - Which training data is most important in a particular scenario and how much is needed to improve performance?

\section{CONTEXT-AWARE SYSTEM MODEL}

In this section, we introduce the $\mathrm{C} 2 \mathrm{D} 2$ system that improves the performance of vehicular networks by using context information. Making applications "context-aware" has been a popular research topic since the early 1990s [1] where the emphasis is on gathering data from sources such as sensors and interpreting them into a smaller set of predefined human-centric context. Our work considers the relationship between a large amount of data 
collected from automotive sensors and other external sources which is resolved into a set of contexts and fed into a decision engine to decide a parameter set for each layer of the protocol stack. The fundamental thesis of our work is that the context of vehicles leads to a particular wireless behavior and enabling awareness of such context can provide vastly improved performance. Other works have begun to explore context awareness for vehicular networks [2-7]. When considering the use of context, the vehicular communication requirements must be carefully understood. These requirements change based on many aspects, including but not limited to vehicle location (city/suburban/rural), application priorities (safety, non-safety), and vehicle speed. Here, we propose an architecture that leverages information from vehicle sensors along with feedback from network protocols at all layers of the stack to efficiently adapt transmission parameters. Fig. 1 shows our system design with three main parts enabling context awareness: collection, decision, and distribution. Tens of vehicular sensors provide information on various things such as location, speed, temperature, and humidity. One part of our system, collection, deciphers the context information that is useful for improving wireless performance from a number of other pieces of data. The relevant sensing information is then passed to two different blocks: to the decision engine for feeding improved network layer choices to the existing network stack and to the database to be stored with network performance data for online training of the system.

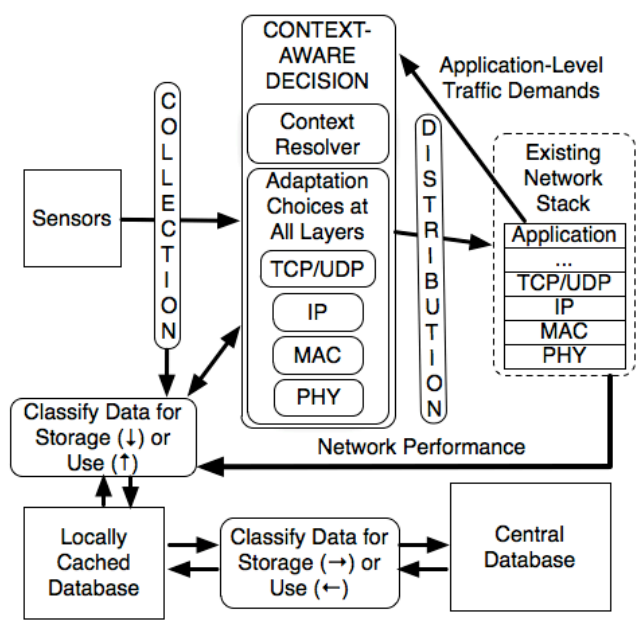

Figure 1. Context-aware Collection, Decision, and Distribution (C2D2) system diagram.

The decision engine will first resolve one environment from another (i.e., a context resolver). For this context, there are different adaptation choices that could be made per network stack layer. Primarily, the decision engine will attempt to eliminate poor choices per layer, leaving the best choices from which the existing network stack will choose. For example, the highest modulation order may never be appropriate in a downtown setting as strong multipath can preclude such a transmission scheme. Each layer has both good and poor choices and that context can help lead protocols to better decisions. These types of adaptation choices exist for all layers of the network stack. Multiple dimensions exist for these adaptation choices such as transmit power, frequency, antenna type and usage, modulation rate, physical layer coding, transport protocol, and routing scheme with one choice leading to a different choice at other layers. Furthermore, the application requirements impose a structure to the feasible operating region in this multi-dimensional space that satisfies the desired QoS. The decision engine will then distribute the adaptation choices that have been refined by context information to the existing network stack without the need for modification. There will also be some recording of observed network performance to feed into the classification algorithm (along with the current collection and decision data). The classifying algorithm will determine if the data tuple should be used for storage in a locally cached or remotely global database. The decision to store the data hinges on various factors that chiefly include how close the current data tuple differs from the training that has been recorded in the local and/or global database. We make the distinction between the two databases types since acquiring remote training data uses system resources. Imagine, for instance, a global database that has training data for a large number of different contexts and the local database is empty. Even in this case, a tradeoff exists of wireless bandwidth overhead to acquire training from this database and the gains of operating more efficiently. Nonetheless, the online training of a system to different contexts that the vehicle encounters can be done without the need to expend additional bandwidth in many cases. Moreover, a system could cache decisions locally until it has a wired or high-bandwidth wireless connection to upload its training to the global database.

\section{SUMMARY}

Vehicular networks pose challenging environments to maintain connectivity or achieve high levels of throughput due to the dynamic nature of wireless channels and high degree of mobility. In this work, we discussed a framework which addresses the adaptation problem via context-aware collection, decision, and distribution (C2D2) to all layers of the network stack. The C2D2 engine will work in parallel to existing protocols by offering the adaptation options that have historically satisfied the application specified QoS levels in a particular context. We are currently developing an automated measurement system that allows synchronization of sensor and network performance data. We are also creating a database populated with data from various measurements both emulated and measured in the field.

\section{REFERENCES}

[1] M. Baldauf, S. Dustdar, and F. Rosenberg, "A survey on context-aware systems," International Journal of Ad Hoc and Ubiquitous Computing, vol. 2, no. 4, pp. 263-277, 2007.

[2] A. Torok, P. Laborczi, and B. Mezny, "Context-aware channel coordination for DSRC," in Pervasive Systems, Algorithms, and Networks, Dec. 2009.

[3] J. Santa and A. Gomez-Skarmeta, "Sharing context-aware road and safety information," IEEE Pervasive Computing, vol. 2009 , no. 1 , pp. 58-65, 2009.

[4] Z. Wang and M. Hassan, "Context-aware channel coordination for DSRC," in IEEE GLOBECOM Workshops, NewOrleans, LA, Dec. 2008.

[5] P. Shankar, T. Nadeem, J. Rosca, and L. Iftode, "CARS: Context-aware rate selection for vehicular networks," in IEEE ICNP, Orlando, Florida, Oct. 2008.

[6] V. Dumitrescu and J. Guo, "Context assisted routing protocols for inter-vehicle wireless communication," in IEEE Intelligent Vehicles Symposium, June 2005.

[7] O. Mehani, R. Boreli, and T. Ernst, "Context-adaptive vehicular network optimization," in International Conference on Intelligent Transport Systems Telecommunications, Lille, France, Oct. 2009. 